\title{
Biodegradation of organonitriles by adapted activated sludge consortium with acetonitrile-degrading microorganisms
}

\author{
Tinggang $\mathrm{Li}^{a, b}$, Junxin Liu ${ }^{b}$, Renbi Bai ${ }^{\mathrm{c}, *}$, Dieudonne-Guy Ohandja ${ }^{d}$, Fook-Sin Wong ${ }^{a}$ \\ anstitute of Environmental Science and Engineering, Nanyang Technological University, Singapore 637723, Singapore \\ ${ }^{b}$ Research Center for Eco-Environmental Sciences, Chinese Academy of Sciences, Beijing 100085, People's Republic of China \\ ${ }^{c}$ Division of Environmental Science and Engineering, National University of Singapore, Singapore 117576, Singapore \\ ${ }^{\mathrm{d} C e n t r e ~ f o r ~ E n v i r o n m e n t a l ~ P o l i c y, ~ I m p e r i a l ~ C o l l e g e ~ L o n d o n, ~ P r i n c e ~ C o n s o r t ~ R o a d, ~ L o n d o n ~ S W 7 ~ 2 A Z, ~ U K ~}$
}

\section{A R T I C L E I N F O}

\section{Article history:}

Received 30 January 2007

Received in revised form

6 April 2007

Accepted 30 April 2007

Available online 8 May 2007

Keywords:

Biodegradation

Organonitriles

Activated sludge consortium

Adaptation

Degradation pathway

\begin{abstract}
A B S T R A C T
A microbial process for the degradation of three types of structurally distinct organonitriles (i.e., saturated and unsaturated aliphatic nitrile and aromatic nitrile) was studied. Microorganisms were enriched from the activated sludge of a pharmaceutical wastewater treatment plant and adapted through providing acetonitrile as the sole carbon and nitrogen source for their growth. The adapted mixed culture was then examined for their capability of degrading acetonitrile, acrylonitrile and benzonitrile under various operational conditions. The performance of biodegradation and the metabolic intermediate- and end-products in the process were monitored. The results show that an average removal rate of $0.083 \mathrm{~g}$ acetonitrile $\mathrm{g}^{-1}-\mathrm{VSSh}^{-1}, 0.0074 \mathrm{~g}$ acrylonitrile $\mathrm{g}^{-1}-\mathrm{VSSh}^{-1}$ or $0.0029 \mathrm{~g}$ benzonitrile $\mathrm{g}^{-1}$-VSS $^{-1}$ was achieved in the batch bioreactor under the common operational condition of $25^{\circ} \mathrm{C}$ and $\mathrm{pH}$ 7. The biodegradation of acetonitrile and acrylonitrile showed a two-step pathway, with the generation of acetamide followed by acetic acid and ammonia for acetonitrile or acrylamide followed by acrylic acid and ammonia for acrylonitrile. However, the biodegradation of benzonitrile appeared to have only one step, with the direct production of benzoic acid and ammonia, but without benzamide being detected in the process. The results suggest that, depending on the substrates, the adapted mixed culture can develop very different degradation pathways, such as nitrile hydratase plus amidase for acetonitrile or acrylonitrile and nitrilase for benzonitrile. Therefore, the adapted mixed culture has a great potential and flexibility for actual applications in biodegradation of various organonitrile compounds.
\end{abstract}

(c) 2007 Elsevier Ltd. All rights reserved.

\section{Introduction}

Organonitriles represent a class of extremely toxic compounds (and priority pollutants) that affect various levels of lives, including human beings, and are known to be highly carcinogenic and mutagenic (Ahmed and Farooqui, 1982; Johammsen et al., 1986; Nawaz et al., 1989). Typical examples of these compounds include acetonitrile, acrylonitrile and benzonitrile that are widely used in laboratories and industries as solvents and extractants, or used as an ingredient in pharmaceuticals, plastics, synthetic rubbers, drug intermediates (chiral synthons), herbicides and pesticides (e.g., dichlobenil, bromoxynil,

*Corresponding author. Tel.: +656516 4532; fax: +65 67744202 .

E-mail address: esebairb@nus.edu.sg (R. Bai).

0043-1354/\$ - see front matter (c) 2007 Elsevier Ltd. All rights reserved.

doi:10.1016/j.watres.2007.04.033 
ioxynil, buctril), etc. (Henahan and Idon, 1971; Banerjee et al., 2002; Kao et al., 2006). As a consequence, organonitrile compounds are often contained in the effluents from these applications and thus present a challenge in the decomposition/ detoxification of these compounds in wastewater treatment. It is therefore of great research and practical interest in investigating technologies/processes that can effectively treat these compounds before their safe discharge into the environment or mixing with other wastewater for further treatment.

Although chemical methods may be available for treating these pollutants, such as ozone and photocatalytic oxidation, the harsh reaction conditions, the generation of secondary pollutants and the high operational cost associated with these methods have often made these methods not a desirable choice (Adjei and Ohta, 1999; Nagle et al., 1995). Bioremediation, an environmentally friendly technology, has been demonstrated to have the prospect to eliminate these compounds by degrading them into harmless intermediates or, ultimately, carbon dioxide and water (Nawaz et al., 1991; Håkansson et al., 2005). In this direction, microbial degradation of organonitrile compounds (and their derivatives) has been extensively explored by many researchers using different isolates, such as Brevibacterium imperalis CBS489-74 (Alfani et al., 2001), Candida guilliermondii CCT 7202 (Dias et al., 2001), Comamonas testosteroni and Acidovorax sp. (Wang et al., 2004), Corynebacterium sp. C5 (Yamamoto et al., 1992), Cryptococcus sp. UFMG-Y28 (Rezende et al., 2000), Klebsiella oxytoca (Kao et al., 2006), Kluyveromyces thermotolerans MGBY 37 (Prasad et al., 2005), Nocardia rhodochrous (DiGeronimo and Antoine, 1976), Paracoccus thiophilus (Lee and Wang, 2004), Pseudomonas marginalis (Babu et al., 1995), Pseudomonas putida (Nawaz et al., 1989), Rhodococcus erythropolis A10 (Acharya and Desai, 1997), R. erythropolis BL1 (Langdanhl et al., 1996), Rhodococcus rhodochrous PA-34 (Bhalla et al., 1992) and Rhodococcus sp. N 774 (Endo and Watanabe, 1989). However, in spite of the fact that an isolated species of microorganisms may show a promising potential in degrading these toxic organonitrile compounds, it is probably of greater practical importance to be able to use a mixed culture (a whole consortium rather than one single species), if possible, for the remediation or degradation of these toxic compounds. To the best of our knowledge, information on biodegradation of organonitrile compounds with a mixed culture has been very limited in the literature. In addition, many other factors, including $\mathrm{pH}$, initial substrate concentration, process temperature and oxygen content, may also affect the biodegradation performance. While biodegradation of organonitriles has been commonly carried out under aerobic condition, there have been only a few studies reported in anaerobic decomposition of acetonitrile by a single species, concluding that the process was rather slow and severely limited by the acetonitrile toxicity (Nagle et al., 1995; Muñoz et al., 2005). So far, not much information is available on the anaerobic degradation of acetonitrile or any other organonitrile compounds by a mixed culture.

In the present study, we investigated the performance and efficiency of biodegradation of three typical types of organonitrile compounds in batch bioreactors with adapted activated sludge consortium as the inoculum. The objective is to examine the possibility of decomposing organonitriles in an environment similar to activated sludge wastewater treatment. The study began with acetonitrile degradation with a mixed culture adapted with acetonitrile-utilizing microorganisms. The process was then expanded to other substrates, i.e. the degradation of unsaturated (acrylonitrile) and aromatic (benzonitrile) organonitriles with the acetonitrile-adapted mixed culture. The biotransformation pathways of the organonitrile compounds by the adapted activated sludge consortium were also discussed.

\section{Materials and methods}

\subsection{Cultivation of activated sludge consortium as bioreactor inoculum}

Raw activated sludge with total suspended solid (TSS) and volatile suspended solid (VSS) of 13.37 and $9.62 \mathrm{gL}^{-1}$, respectively, was obtained from a pharmaceutical wastewater treatment plant in Singapore. The mixed culture of microorganisms in the sludge was then acclimated with acetonitrile as the sole carbon and nitrogen source to adapt the mixed culture with acetonitrile-degrading capability. Selective enrichment and cultivation of the microorganisms was conducted in a 21 glass bottle bioreactor containing 11 of a synthetic mineral salt medium (MSM) solution with the composition of (per liter): $500 \mathrm{mg} \mathrm{KH}_{2} \mathrm{PO}_{4}, 1100 \mathrm{mg} \mathrm{Na} \mathrm{N}_{2} \mathrm{H}$ $\mathrm{PO}_{4} \cdot 2 \mathrm{H}_{2} \mathrm{O}, 18 \mathrm{mg} \mathrm{CaCl} \cdot 2 \mathrm{H}_{2} \mathrm{O}, 23 \mathrm{mg} \mathrm{MgCl}_{2} \cdot 6 \mathrm{H}_{2} \mathrm{O}, 26 \mathrm{mg}$ $\mathrm{MnCl}_{2} \cdot 2 \mathrm{H}_{2} \mathrm{O}, 75 \mathrm{mg} \mathrm{FeCl} \cdot 4 \mathrm{H}_{2} \mathrm{O}, 2.1 \mathrm{mg} \mathrm{NaVO}$, $1.7 \mathrm{mg} \mathrm{Na} \mathrm{Na}_{2}$ $\mathrm{WO}_{3} \cdot 2 \mathrm{H}_{2} \mathrm{O}, 4.9 \mathrm{mg} \mathrm{H}_{3} \mathrm{BO}_{3}, 3.8 \mathrm{mg} \mathrm{NiSO} \cdot 6 \mathrm{H}_{2} \mathrm{O}, 2.1 \mathrm{mg} \mathrm{ZnCl}$, $1.8 \mathrm{mg} \mathrm{CoCl}, 2.1 \mathrm{mg} \mathrm{Na} \mathrm{MoO}_{4} \cdot 2 \mathrm{H}_{2} \mathrm{O}$ and $2.9 \mathrm{mg} \mathrm{CuSO}{ }_{4} \cdot 2 \mathrm{H}_{2} \mathrm{O}$; plus acetonitrile substrate at the concentration of $300-700 \mathrm{mgL}^{-1}$ as the sole carbon and nitrogen sources. Then, the bioreactor was inoculated with 11 of the raw activated sludge and incubated at room temperature $\left(25^{\circ} \mathrm{C}\right)$ and stirred at $180 \mathrm{rpm}$ on a rotary shaker for a period of up to 2 months. The adapted microbial consortium or mixed culture was washed thrice with freshly prepared MSM solution and then resuspended in a fresh MSM solution to obtain the inoculum (with TSS $6.38 \mathrm{gL}^{-1}$ and VSS $5.71 \mathrm{gL}^{-1}$ ) used in the biodegradation study for acetonitrile, acrylonitrile or benzonitrile. All the chemicals in the study were of at least analytical-grade purity and were purchased from Sigma-Aldrich, USA.

\subsection{Biodegradation experiments}

The toxicity or inhibition of acetonitrile to the adapted microbial consortium was first assessed. A number of 250$\mathrm{mL}$ serum bottles was each filled with $117 \mathrm{~mL}$ of the MSN solution and then inoculated with $63 \mathrm{~mL}$ of the adapted microbial culture with acetonitrile-degrading microorganisms to prepare the batch bioreactors that had a VSS of $2 \mathrm{gL}^{-1}$ and an initial acetonitrile concentration from 0.5 to $10 \mathrm{gL}^{-1}$, respectively. To ensure the same initial oxygen condition, the oxygen entrapped in the bioreactors (in the headspace of the serum bottles and in the bulk liquid) was first removed by sparging them with a gas mixture containing $70 \%$ nitrogen and $30 \%$ carbon dioxide. The initial $\mathrm{pH}$ values of the contents in the batch bioreactors were all adjusted to 7.0 using $1 \mathrm{M}$ 
$\mathrm{H}_{2} \mathrm{SO}_{4}$. The serum bottle batch bioreactors were then sealed with aluminum caps with PTFE/silicone septum and the bioreactors were incubated at room temperature $\left(25^{\circ} \mathrm{C}\right)$ and stirred at $180 \mathrm{rpm}$ on a rotary shaker. Control tests without acetonitrile were also performed under the identical conditions. Oxygen of $20 \mathrm{~mL}$ ( $1 \mathrm{~atm}$ ) was regularly added into the serum bottles with a glass syringe. Liquid samples of $2 \mathrm{~mL}$ were periodically taken from each batch bioreactor to monitor the concentrations of acetonitrile in the bioreactors.

Then, acetonitrile biodegradation experiments with the adapted mixed culture were performed in a number of 250-mL serum bottle batch bioreactors. They were each filled with $117 \mathrm{~mL}$ of the MSM solution and inoculated with $63 \mathrm{~mL}$ of the adapted microbial culture and all the bioreactors (with a VSS of $2 \mathrm{gL}^{-1}$ ) had the same initial acetonitrile concentration of $2 \mathrm{gL}^{-1}$. The experiments were conducted under various initial $\mathrm{pH}$ values (3-11), temperatures $\left(10,20,25,35\right.$ and $\left.50^{\circ} \mathrm{C}\right)$, and under aerobic or anaerobic conditions. Initially, the batch bioreactors were sparged with a nitrogen gas to remove any oxygen retained in the headspace and bulk liquid of the serum bottles. The initial $\mathrm{pH}$ values of the contents in the batch bioreactors were adjusted, using $1 \mathrm{M} \mathrm{H}_{2} \mathrm{SO}_{4}$, to the various desired levels. The serum bottles were then sealed with aluminum caps with PTFE/silicone septum and incubated at the desired temperature and stirred at $180 \mathrm{rpm}$ in an incubator shaker. Sterile control tests (without biomass) to record the abiotic loss of acetonitrile were also carried out under the same conditions. Oxygen of $20 \mathrm{~mL}$ (1 atm) was regularly added into each of the serum bottles with a glass syringe in the aerobic biodegradation experiments (dissolved oxygen (DO) concentration was maintained at about $2-2.3 \mathrm{mg} / \mathrm{L}^{-1}$ ) but no oxygen was added in the anaerobic biodegradation experiments. Samples of $2 \mathrm{~mL}$ were taken with a glass syringe at various time intervals to analyze the biomass (in terms of VSS), pH, inorganic carbon (IC), as well as the contents of acetonitrile, acetamide, acetic acid, ammonia, nitrite, nitrate, etc. Similar experiments for acrylonitrile and benzonitrile biodegradation were also conducted as described above. Except the experiments for the effects of temperature and $\mathrm{pH}$ values, all other experiments were conducted at a typical ambient temperature of $25^{\circ} \mathrm{C}$ and under the neutral $\mathrm{pH}$ of 7 for the reason of practical application consideration.

\subsection{Sample analyses}

The TSS and VSS were determined at $105^{\circ} \mathrm{C}(4 \mathrm{~h})$ and $550^{\circ} \mathrm{C}$ $(2 \mathrm{~h})$, respectively (APHA, 1998). The $\mathrm{pH}$ values and DO concentrations were measured by a $\mathrm{pH}$ meter (YSI 100, USA) and a DO meter (YSI200, USA), respectively. For other analyses, the samples were first centrifuged at $13,200 \mathrm{~g}$ at $4{ }^{\circ} \mathrm{C}$ for $20 \mathrm{~min}$, and then the supernatants were used. Acetonitrile, acrylonitrile, benzonitrile and their degraded metabolic intermediate or end products (e.g. amides, carboxylic acids, etc.) were analyzed by gas chromatography (Agilent 6890N, Agilent, USA) equipped with an Agilent HP-5 $5 \%$ phenyl siloxane capillary column $(30 \mathrm{~m} \times 320 \mu \mathrm{m}$ internal diameter $\times 0.25 \mu \mathrm{m}$ film thickness) and a flame ionization detector. The injector was kept at $250^{\circ} \mathrm{C}$, the column was programmed from 35 to $250^{\circ} \mathrm{C}$ at $15^{\circ} \mathrm{C} \mathrm{min}^{-1}$ increments ( 3 min hold), and the detector was set at $250^{\circ} \mathrm{C}$. Helium was used as the carrier gas at $7 \mathrm{~mL} \mathrm{~min}^{-1}$. The sample injection volume was $1 \mu \mathrm{L}$, and the flow rates of hydrogen and air were 40 and $450 \mathrm{~mL} \mathrm{~min}^{-1}$, respectively. IC was analyzed with a TOC/TN-VCSH analyzer (Shimadhu, Japan). Ammonia, nitrite and nitrate were determined through the Hach test kits with a UV-vis Spectrophotometer (DR 5000, Hach, USA).

\section{Results and discussion}

\subsection{Acetonitrile inhibition effect}

The influence of initial acetonitrile concentrations on the inhibition of the adapted mixed culture was investigated at concentrations of $0.5,1,2,3,5,7$ and $10 \mathrm{gL}^{-1}$. Fig. 1 shows the experimental results obtained. It has been found that the presence of acetonitrile in the mixed culture medium at a concentration of $5 \mathrm{gL}^{-1}$ or below had no noticeable effect on the inhibition. There was no lag phase and acetonitrile was degraded immediately from the beginning of the process. As shown in Fig. 1, a complete reduction in acetonitrile concentration was achieved at about $10 \mathrm{~h}$ in the case of initial concentration of $1 \mathrm{gL}^{-1}$ or at about $75 \mathrm{~h}$ for the initial concentration of $5 \mathrm{gL}^{-1}$. When the concentration of acetonitrile in the mixed culture medium increased to $7 \mathrm{gL}^{-1}$ or higher, the inhibition effect began to be observed in the experiments. For example, at the initial concentration of $10 \mathrm{gL}^{-1}$, only $7.4 \mathrm{gL}^{-1}$ of the acetonitrile was degraded at $186 \mathrm{~h}$ and the process showed an initial lag phase of $4 \mathrm{~h}$ (see Fig. 1), suggesting that significant inhibitive effect on the acetonitrile-degrading microorganisms occurred, at least initially, in the bioreactor. It was found that a significant amount of ammonia was accumulated in the bioreactor in this case, which may result in further inhibition effect on the microbial activity (Lee et al., 2000). Nevertheless, a 76\% of reduction in the acetonitrile concentration was still obtained at the end of

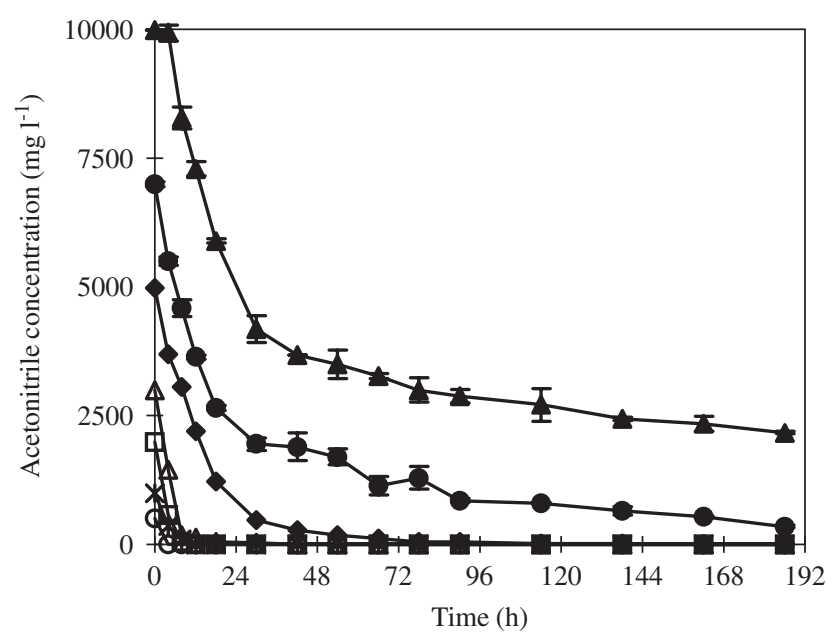

Fig. 1 - Changes of concentration with process time from inhibition experiments at various initial acetonitrile concentrations: $(\odot) 0.5 \mathrm{~g} \mathrm{~L}^{-1}$, ( $\left.^{*}\right) 1 \mathrm{~g} \mathrm{~L}^{-1},(\square) 2 \mathrm{~g} \mathrm{~L}^{-1},(\triangle) 3 \mathrm{~g} \mathrm{~L}^{-1}$, $(\diamond) 5 \mathrm{~g} \mathrm{~L}^{-1},(\bullet) 7 \mathrm{~g} \mathrm{~L}^{-1}$ and $(\Delta) 10 \mathrm{~g} \mathrm{~L}^{-1}$ (vertical bars represent the deviation calculated from two duplicates), $25^{\circ} \mathrm{C}$ and $\mathrm{pH} 7$. 
the inoculation even though at such a high initial acetonitrile concentration of $10 \mathrm{~g} \mathrm{~L}^{-1}$ in the bioreactor. The results in Fig. 1 reveal an interesting fact that the adapted mixed culture actually displayed much higher resistance to the toxic organic compound, as compared to single species that were reported to show inhibition at the maximum concentration of $1 \mathrm{gL}^{-1}$ for acetonitrile (Nagle et al., 1995). Muñoz et al. (2005) also found that, when acetonitrile was present at $1 \mathrm{gL}^{-1}$, the activity of Chlorella sorokiniana decreased by $18 \%$, that of Scenedesmus obiquus and Selenastrum capricornutum by $59 \%$ and $36 \%$, respectively, and Chlorella vulgaris was unable to grow at an acetonitrile concentration of 5 or $10 \mathrm{gL}^{-1}$.

The above experimental results demonstrate that the adapted mixed culture was capable of using acetonitrile as the sole carbon and nitrogen source for their growth in a wide range of acetonitrile initial concentrations. The apparent specific growth rates of the adapted mixed culture under the various initial acetonitrile concentrations during the incubation are shown in Fig. 2. It is clear that the specific growth rate, in terms of $\mu_{\max }$, increased almost linearly with the initial acetonitrile concentration $\left(C_{0}\right)$ at $C_{0}$ below $2 \mathrm{gL}^{-1}$, reached the highest $\left(0.37 \mathrm{~h}^{-1}\right)$ at $\mathrm{C}_{0}=2-3 \mathrm{gL}^{-1}$, and then gradually decreased, with the further increase of the initial acetonitrile concentrations, to a level of about $0.11 \mathrm{~h}^{-1}$ at $C_{0}=10 \mathrm{gL}^{-1}$. All the growth rates are in positive values, which provide evidence that the adapted mixed culture degraded acetonitrile and used it as the nutrients for their growth in these experiments. At low acetonitrile concentrations, since there was no inhibition effect, the increase of acetonitrile concentration provided more available nutrients for the microorganisms and therefore stimulated a greater increase in the growth rate. At high acetonitrile concentrations, however, the inhibition effect of acetonitrile may have greatly compromised the effect of acetonitrile as the effective nutrient source for the microorganisms. As a result, the apparent growth rate decreased with the increase of the acetonitrile concentrations in the high concentration range. Clearly, the results in Fig. 2 show that there is an optimum

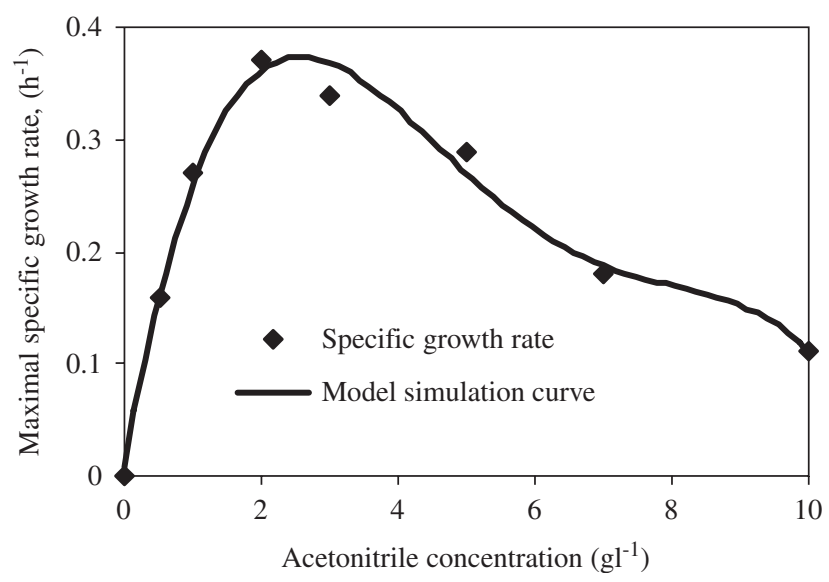

Fig. 2 - Specific growth rates of the mixed culture versus initial acetonitrile concentrations (specific growth rate $(\mu)$ was derived from the mixed culture VSS in the exponential cell growth phase), $25^{\circ} \mathrm{C}$ and $\mathrm{pH} 7$. acetonitrile loading concentration at which the growth rate of the mixed culture (hence the degradation rate of acetonitrile) in the bioreactor is the highest. The removal rate of acetonitrile at the highest growth rate is calculated to be 0.083 g acetonitrile $\mathrm{g}^{-1}-\mathrm{VSSh}^{-1}$ or $4 \mathrm{gL}^{-1} \mathrm{~d}^{-1}$. This removal rate appears to be much better than those reported in the literature. For example, Muñoz et al. (2005) obtained acetonitrile degradation rates at up to 1.9 or $2.3 \mathrm{gL}^{-1} \mathrm{~d}^{-1}$ in 565 and $545 \mathrm{~mL}$ algal-bacterial bioreactors in their study; and Håkansson and Mattiasson (2002) achieved the degradation rate of $2 \mathrm{gL}^{-1} \mathrm{~d}^{-1}$ in a $550 \mathrm{ml}$ continuously stirred biofilm reactor with the microorganism consortium from an activated sludge wastewater plant.

\subsection{Effect of temperature and $\mathrm{pH}$ on acetonitrile biodegradation}

Although most of the experiments in this study were preferentially conducted at an ambient temperature of $25^{\circ} \mathrm{C}$ and the neutral $\mathrm{pH}$ of 7 , the effects of temperature and solution $\mathrm{pH}$ on the biodegradation performance were also examined. Figs. 3 and 4 show the results of biodegradation of acetonitrile with the adapted mixed culture under a wide range of process temperatures and $\mathrm{pH}$ values, respectively. It is evident that the variations in acetonitrile biodegradation by the adapted mixed culture in the temperature and $\mathrm{pH}$ ranges examined were significant (more than 80\%). The optimum temperature and $\mathrm{pH}$ in these cases appeared to be at around $30^{\circ} \mathrm{C}$ and $\mathrm{pH} 7.5$, respectively. Biodegradation of acetonitrile by the adapted mixed culture almost completely stopped at temperatures higher than $50^{\circ} \mathrm{C}$ or lower than $10^{\circ} \mathrm{C}$, or at $\mathrm{pH}$ values greater than 11 or smaller than 3 . In spite of this, the adapted mixed culture can still be considered to show relatively high bioactivity for acetonitrile degradation in a wide range of temperature $\left(20-35^{\circ} \mathrm{C}\right)$ and $\mathrm{pH}(4-10)$ and performed the best in the temperature range of $25-30^{\circ} \mathrm{C}$ (with acetonitrile degradation rates of $\left.0.083-0.091 \mathrm{gg}^{-1}-\mathrm{VSS} \mathrm{h}^{-1}\right)$ and

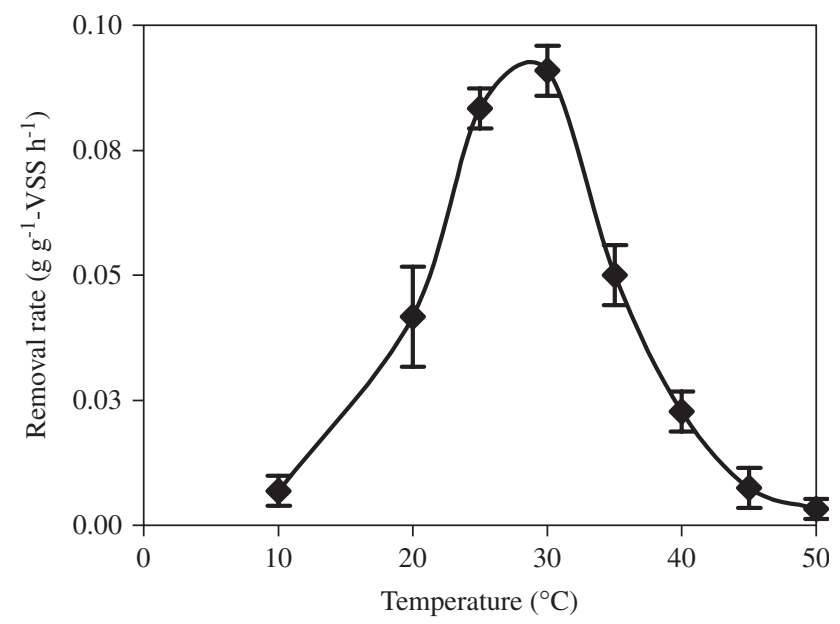

Fig. 3 - Effect of incubation temperature on acetonitrile removal rate in the batch bioreactor (initial acetonitrile in the culture medium: $2 \mathrm{~g} \mathrm{~L}^{-1}$, vSS: $2 \mathrm{~g} \mathrm{~L}^{-1}, \mathrm{pH}=7$, vertical bars represent the deviation calculated from two duplicates). 


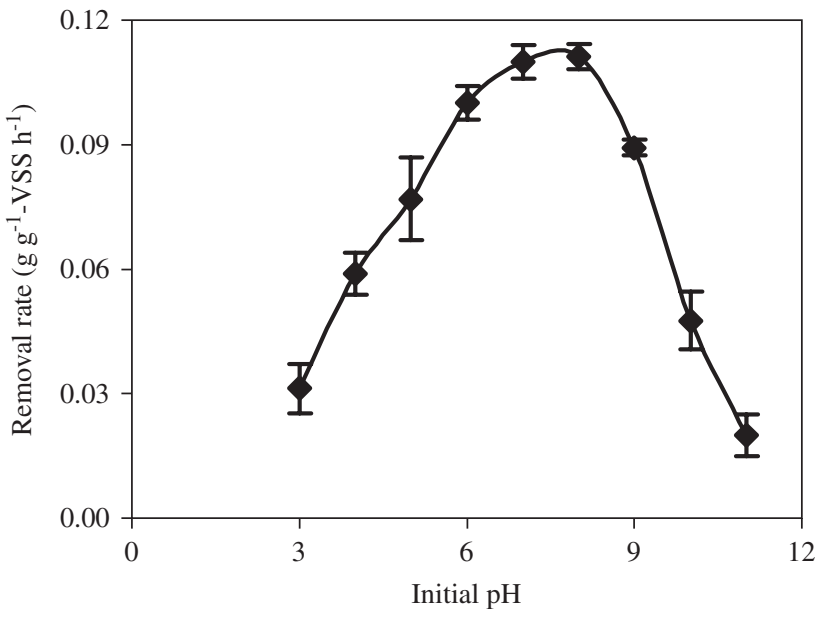

Fig. 4 - Effect of initial $\mathrm{pH}$ on acetonitrile removal rate in the batch bioreactor (initial acetonitrile in the culture medium: $2 \mathrm{~g} \mathrm{~L}^{-1}$, vSS: $2 \mathrm{~g} \mathrm{~L}^{-1}, 25^{\circ} \mathrm{C}$, vertical bars represent the deviation calculated from two duplicates).

in the $\mathrm{pH}$ range of $6-8$ (with acetonitrile degradation rates of $0.100-0.111 \mathrm{gg}^{-1}-\mathrm{VSSh}^{-1}$ ), respectively. For single species, others also noted the existence of an optimum temperature and $\mathrm{pH}$ for acetonitrile biodegradation but a wide variation of these optima with the cultures. Manolov et al. (2005) reported that the optimum $\mathrm{pH}$ and temperature for acetonitrile biodegradation by Comamonas sp. were at pH 7 and $24^{\circ} \mathrm{C}$, respectively, and observed that no signification bioactivity existed at $\mathrm{pH}$ values greater than 8.5 and at temperatures higher than $37^{\circ} \mathrm{C}$ or lower than $14^{\circ} \mathrm{C}$. Bhalla et al. (1992) reported that the $\mathrm{pH}$ and temperature optima by $\mathrm{R}$. rhodochrous were at pH 7.5 and $35^{\circ} \mathrm{C}$. Therefore, the adapted mixed culture has greater tolerance in the variation of temperature and solution $\mathrm{pH}$.

\subsection{Metabolic products of acetonitrile biodegradation under aerobic condition}

The biodegradation of acetonitrile with the adapted mixed culture in the aerobic process generated the metabolic products of acetamide, acetic acid and ammonia in the culture medium (see Fig. 5). The reduction of acetonitrile was $71.3 \%$ at $4 \mathrm{~h}$ and $97.3 \%$ at $8 \mathrm{~h}$, respectively. After $12 \mathrm{~h}$ of incubation, no acetonitrile was detected in the culture medium. Consequently, the concentration of the metabolic intermediate product, i.e., acetamide, increased rapidly and accumulated with the reduction of acetonitrile concentration in the culture medium and reached a maximum accumulation of $1804.1 \mathrm{mgL}^{-1}$ at $8 \mathrm{~h}$. Then, with the complete reduction or degradation of acetonitrile, acetamide concentration started to decrease, possibly due to the degradation of acetamide by the microorganisms, and the concentration rapidly decreased to nondetectable at about $30 \mathrm{~h}$. The concentration of acetic acid gradually increased from the beginning and reached a maximum at around $30 \mathrm{~h}$. After that, the concentration decreased gradually to zero at $54 \mathrm{~h}$. The metabolic product of ammonia was found to increase during the entire period of incubation studied and reached

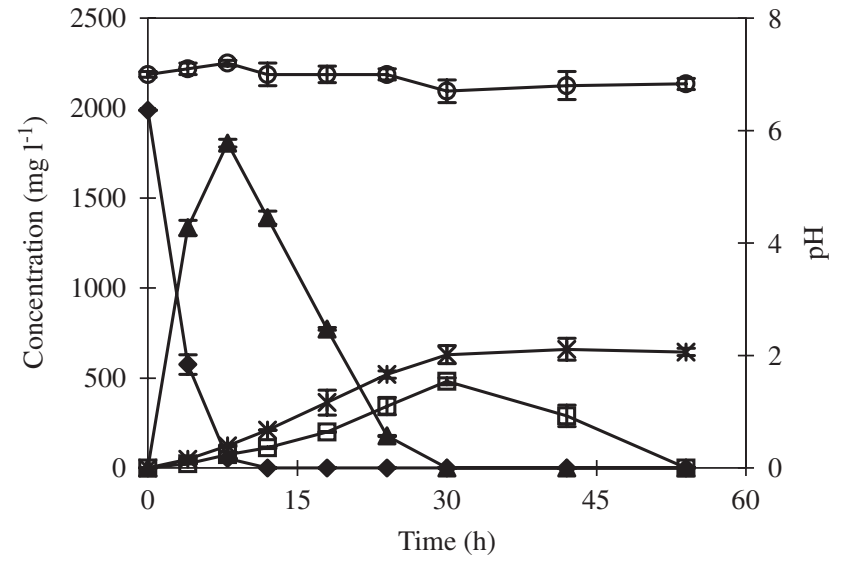

Fig. 5 - Profiles of acetonitrile biodegradation and metabolic intermediate- and end-product formation in the culture medium at $25^{\circ} \mathrm{C}$. ( $\left.\bullet\right)$ Acetonitrile, $(\Delta)$ acetamide, $(\square)$ acetic acid, (*) ammonia, $(\circ) \mathrm{pH}$ (vertical bars represent the deviation calculated from two duplicates).

$645.3 \mathrm{mgL}^{-1}$ at $54 \mathrm{~h}$ of the incubation. The $\mathrm{pH}$ value of the culture medium did not seem to change significantly although a slight decrease was observed in the process. The $\mathrm{pH}$ results are different from that of Acharya and Desai (1997), who reported an increase in $\mathrm{pH}$ value up to $\mathrm{pH} 9$ after $96 \mathrm{~h}$ incubation in a single species system. The relatively constant $\mathrm{pH}$ in the process in this study may be explained by the fact that high buffer capacity was produced by the IC, i.e., $\mathrm{CO}_{2} / \mathrm{HCO}_{3}^{-}$generated at about $1078 \mathrm{mgL}^{-1}$ in the process. The formation of an $\mathrm{NH}_{4} \mathrm{Ac}$-HAc buffer system reduced the effect of ammonia accumulation on the possible increase of the solution $\mathrm{pH}$ value in the culture medium. No nitrite and nitrate were detected during the entire incubation process. In addition, the growth of the mixed culture was evidenced by the increase of VSS in the bioreactor with the production of the metabolic products: acetamide, acetic acid and ammonia in the culture medium (data not shown).

\subsection{Acrylonitrile biodegradation with the adapted mixed culture}

The mixed microbial culture acclimated with acetonitrile as the carbon and nitrogen source was also studied for the biodegradation of acrylonitrile (an example of the unsaturated organonitriles). Fig. 6 displays the typical performances of the bioreactor under aerobic condition in the study. In comparison with those results shown in Fig. 5 for acetonitrile, the results for acrylonitrile in Fig. 6 show somewhat very similar characteristic changes in the process performance. However, the biodegradation of acrylonitrile by the adapted mixed culture appeared to be much slower and took about $140 \mathrm{~h}$ for complete degradation (in contrast to $12 \mathrm{~h}$ for acetonitrile). Acrylamide was generated and its concentration increased from zero at the beginning to a maximum of $1960 \mathrm{mgL}^{-1}$ at $140 \mathrm{~h}$ and then decreased rapidly to zero at $232 \mathrm{~h}$. The removal rate of 0.0074 gacrylonitrile $^{-1}-\mathrm{VSS} \mathrm{h}^{-1}$ was achieved in the bioreactor. The biomass (in terms of VSS) was found to increase continuously in the process 


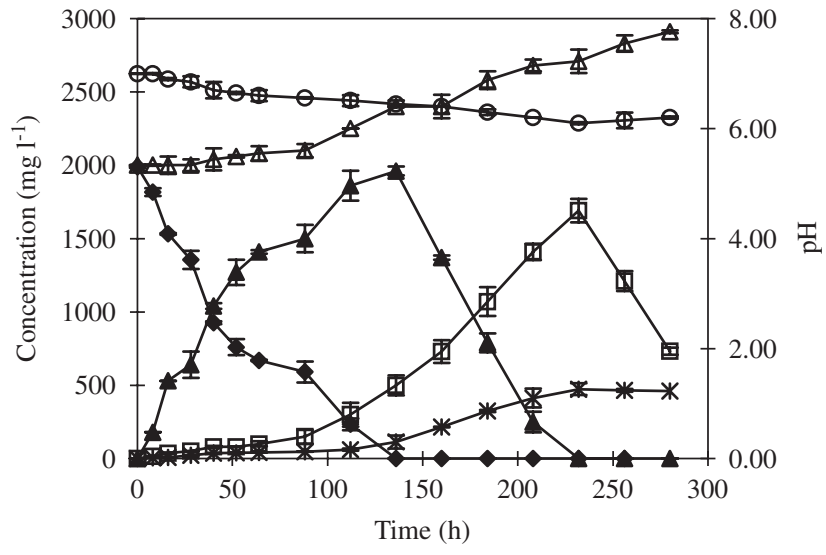

Fig. 6 - Profiles of acrylonitrile biodegradation and metabolic intermediate- and end-product formation in the culture medium at $25^{\circ} \mathrm{C}$. ( $\left.\diamond\right)$ Acrylonitrile, $(\Delta)$ acrylamide, $(\square)$ acrylic acid, (*) ammonia, $(\odot) \mathrm{pH},(\triangle)$ VSS (vertical bars represent the deviation calculated from two duplicates).

(there was no lag phase) and the metabolic products of acrylamide, acrylic acid and ammonia were produced consequently and sequentially in the culture medium. The results indicate that the adapted mixed culture utilized acrylonitrile and then acrylamide in their growth in the process. These results are different from those reported in the literature where single strains, such as C. testosteroni and Pseudomonas sp., for acrylonitrile biodegradation were found to utilize only the metabolic products of acrylic acid and ammonia as the carbon and nitrogen sources (Wang et al., 2004; Shanker et al., 1990). The maximum accumulation of ammonia in the process occurred at $232 \mathrm{~h}$ of the incubation when acrylamide was completely degraded or disappeared. Also, no nitrite and nitrate were detected in the culture medium. The acrylic acid concentration reached a maximum accumulation of $1690.3 \mathrm{mgL}^{-1}$ at $232 \mathrm{~h}$. Again, the $\mathrm{pH}$ in the process did not change significantly, similar to that in the acetonitrile biodegradation experiment discussed earlier. The results confirm that acrylonitrile can be degraded by the mixed culture adapted with acetonitrile as the carbon and nitrogen sources. The results also suggest that the biodegradation of acetonitrile and acrylonitrile by the adapted mixed culture probably followed similar reaction pathways.

\subsection{Benzonitrile biodegradation with the adapted mixed} culture

Similarly, to explore the substrate specificity, the mixed culture adapted with acetonitrile as the carbon and nitrogen sources was examined for the capability of degrading and utilizing benzonitrile (an example of the aromatic organonitriles) as the substrate for their growth. The results on the concentration changes of benzonitrile and the corresponding metabolic products in the process are shown in Fig. 7. A $40 \mathrm{~h}$ lag phase occurred initially and then the concentration of benzonitrile gradually decreased from intial $2 \mathrm{gL}^{-1}$ to
$157.2 \mathrm{mgL}^{-1}$ at $314 \mathrm{~h}$ (the end of the incubation). A removal rate of 0.0029 gbenzonitrile $\mathrm{g}^{-1}-\mathrm{VSS}^{-1}$ by the culture was achieved in the biodegradation process. The growth of the biomass (in terms of VSS increase) also started from $40 \mathrm{~h}$ and the increase continued throughout the rest period of the process examined. The metabolic products of benzoic acid and ammonia were detected in the culture medium after the initial lag phase. The concentrations of benzoic acid and ammonia increased with the reduction of benzonitrile concentration and reached their highest levels at the end of the incubation of $314 \mathrm{~h}$. It is interesting to note that no benzamide was detected during the entire incubation process in this study. Mass balance analysis also confirmed that benzamide was not produced in the culture medium. It appears that the biodegradation of benzonitrile followed a different reaction pathway from that of acetonitrile and acrylonitrile by the adapted mixed culture. In spite of the high toxicity of benzonitrile to the microorganisms, the biodegradation of benzonitrile with the adapted mixed culture was still satisfactorily achieved after a period of cultivation in the environment with only benzonitrile as the sole carbon and nitrogen sources. A $92.1 \%\left(1840 \mathrm{mgL}^{-1}\right)$ of removal or degradation of benzonitrile was actually accomplished at the end of the incubation of $314 \mathrm{~h}$ in this study. The $\mathrm{pH}$ in the culture medium showed similar small changes as in those cases for acetonitrile and acrylonitrile. In the literature, Kao et al. (2006) reported that a $2 \mathrm{mM}$ $\left(206 \mathrm{mgL}^{-1}\right)$ benzonitrile concentration caused significant inhibition effects on the growth of Klebsiella oxytoca and no enzymatic activity of Klebsiella oxytoca was observed in the culture medium with $25 \mathrm{mM}\left(2575 \mathrm{mgL}^{-1}\right)$ acetonitrile or benzonitrile. Nawaz et al. (1992) also reported benzonitrile removal of $865 \mathrm{mg} \mathrm{L}^{-1}$ at $72 \mathrm{~h}$ incubation with the single strain of Klebsiella pneumoniae. These results appear to be less effective than that achieved by the adapted mixed culture in this study.

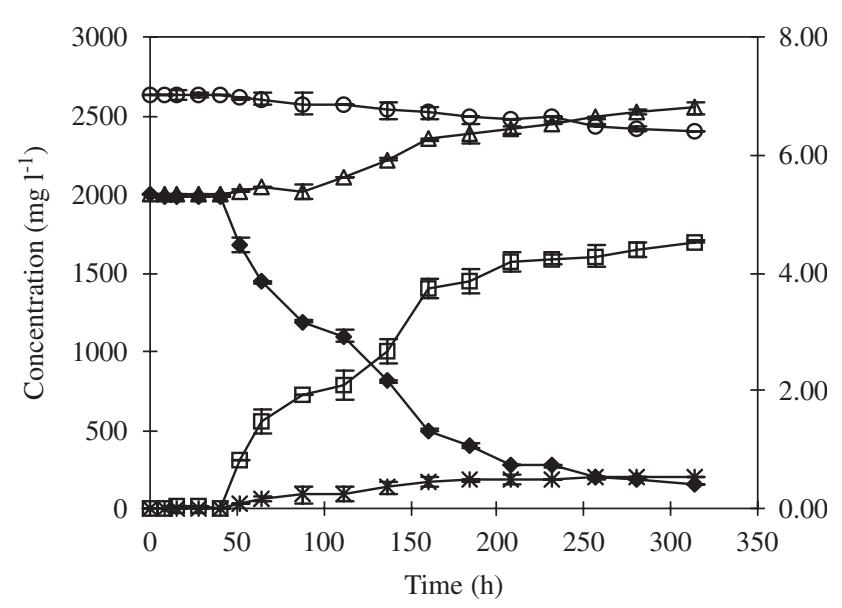

Fig. 7 - Profiles of benzonitrile biodegradation and metabolic product formation in the culture medium at $25^{\circ} \mathrm{C}$. ( $\left.\diamond\right)$ Benzonitrile, $(\square)$ benzoic acid, (*) ammonia, $(\circ)$ pH $(\triangle)$ VSS (vertical bars represent the deviation calculated from two duplicates). 


\subsection{Pathways of organonitrile biodegradation with the adapted mixed culture}

The results from the present study demonstrated that the mixed culture acclimated with acetonitrile is capable of transforming or biodegrading a variety of structurally distinct organonitrile as the sources of nitrogen and carbon for their growth. This indicates that the microorganisms in the mixed culture possess or can develop the necessary mechanisms for the metabolism of different types of organonitriles. The biodegradation performances and the metabolic products, as shown in Fig. 5 for acetonitrile and in Fig. 6 for acrylonitrile, clearly suggest that the processes involve a two-step enzymatic degradation mechanism: the generation of their corresponding amide compounds, followed by further degradation of the amide compounds to the corresponding simple organic acid (acetic acid or acrylic acid) and ammonia. In other words, the nitrile hydratase has converted acetonitrile or acrylonitrile to its respective amides as the intermediate degradation products. Then, the amides were subsequently transformed to their corresponding carboxylic or acrylic acids and ammonia by the amidase. The pathway of the conversion is given in Scheme 1. A similar pathway for the formation of amide, and then carboxylic acid and ammonia from microbial transformation of nitrile compounds was also reported by others (Kao et al., 2006; Rezende et al., 2003). For example, Kao et al. (2006) reported the biodegradation of acetonitrile and propionitrile by Klebsiella oxytoca involving a two-step enzymatic degradation mechanism (no information on the degradation pathway of benzonitrile was given). In contrast, the biodegradation performance and metabolic products of benzonitrile shown in Fig. 7 in this study clearly suggest a one-step reaction pathway, i.e., from benzonitrile directly to benzoic acid and ammonia. No amide (in this case benzamide) was found in the culture medium during the entire incubation process. These results indicate that the microorganisms in the mixed culture acclimated with acetonitriledeveloped nitrilase that directly converted benzonitrile to the metabolic products of benzoic acid and ammonia. The biodegradation pathway of benzonitrile by the adapted mixed culture is also given in Scheme 1. In the literature, both types of nitrile-degradation pathways or mechanisms (i.e., the nitrile hydratase followed by the amidase or the nitrilase only) have been proposed for the microbial metabolism of some nitrile compounds. However, those hypotheses were not well supported by experimental results because the enzymatic activities were derived only from the ammonia product that can liberate from any of the two types of microbial metabolic processes or pathways (Bhalla and Kumar, 2005; Prasad et al., 2005). In the present study, the establishment of the two types of nitrile-degradation pathways was based on the detected metabolic intermediates (if any) and end products in the process, and therefore was more clearly supported by the experimental results. Nitrilases of microbial systems have been known to vary greatly, depending on their affinity toward the types of nitrile substrates. Some of the nitrilases are specific for aliphatic nitriles (Kobayashi et al., 1990), while others for aromatic nitriles (Harper, 1985). In this study, the nitrilase developed by the adapted microorganisms may be specifically for benzonitrile. The present study therefore reveals that the adapted mixed culture can develop very different metabolic pathways according to the nitrile substrates available in the culture medium for their degradation, possibly due to the diverse microbes and enzymes in the mixed culture.

\subsection{Biodegradation under anaerobic condition}

Research in biodegradation of organonitriles under anaerobic condition has been rather limited. It was reported that acetonitrile biodegradation under anaerobic conditions with single strains was very slow and displayed significant inhibition effect due to acetonitrile toxicity, as compared to the performance under aerobic conditions (Nagle et al., 1995; Manolov et al., 2005). As a preliminary exploration, the influence of the anaerobic condition on the biodegradation of acetonitrile with the adapted mixed culture was investigated in this study (under similar conditions as described before for aerobic treatment but without the addition of oxygen into the bioreactor after any initially entrapped oxygen in the bulk liquid and in the headspace of the bottle was removed by sparging it with a nitrogen and carbon
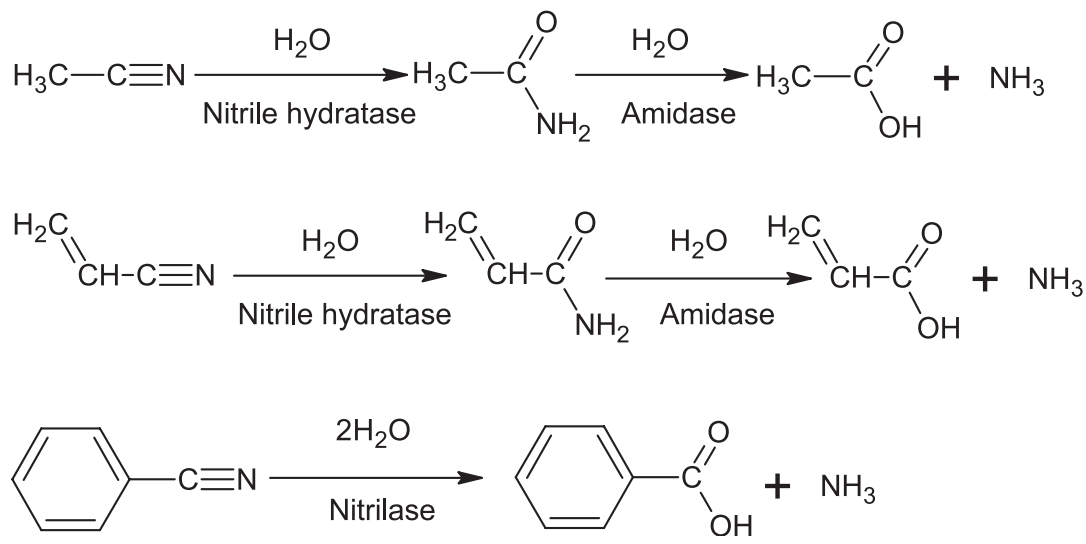

Scheme 1 - Different biodegradation pathways of organonitriles by the adapted mixed culture in this study: (1) for acetonitrile, (2) for acrylonitrile, and (3) for benzonitrile. 


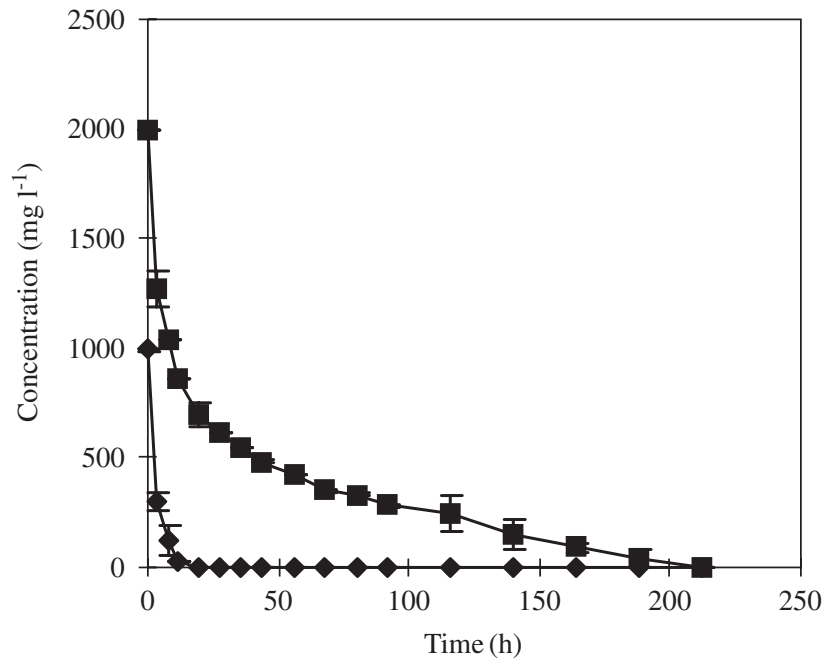

Fig. 8 - Performance of acetonitrile biodegradation under anaerobic condition with the adapted mixed culture. $(\bullet)$ $1 \mathrm{~g} \mathrm{~L}^{-1}$ of acetonitrile, ( $\left.\square\right) 2 \mathrm{~g} \mathrm{~L}^{-1}$ of acetonitrile (vertical bars represent the deviation calculated from two duplicates), initial VSS $=2 \mathrm{~g} \mathrm{~L}^{-1}$, initial $\mathrm{pH} 7$, and $25^{\circ} \mathrm{C}$.

dioxide mixture). Fig. 8 shows some of the typical results on the performance of anaerobic biodegradation of acetonitrile with the adapted mixed culture. At the acetonitrile concentration of $1 \mathrm{gL}^{-1}$, the degradation of acetonitrile was, in fact, fast and the removal reached $69.9 \%$ at $4 \mathrm{~h}$ and $87.7 \%$ at $8 \mathrm{~h}$. The complete removal of acetonitrile was achieved at $20 \mathrm{~h}$, with an average removal rate of $0.025 \mathrm{~g} \mathrm{~g}^{-1}-\mathrm{VSS}^{-1}$. When the acetonitrile concentration was increased to $2 \mathrm{gL}^{-1}$, the biodegradation reaction became much slower. The removal performance was $36.5 \%$ at $4 \mathrm{~h}$ and $48.1 \%$ at $8 \mathrm{~h}$ and the complete removal was achieved at $212 \mathrm{~h}$, with an average removal rate of $0.0047 \mathrm{gg}^{-1}-\mathrm{VSS}^{-1}$. There was no lag phase observed in the process and the metabolic products of acetamide, acetic acid and ammonia were all detected in the culture medium during the incubation. The acetic acid concentration was found to increase with the reaction process time and reached the highest in the culture medium at $212 \mathrm{~h}$ (data not shown). The microorganisms in the mixed culture appeared to be still highly active even though exposed to a $2 \mathrm{gL}^{-1}$ of acetonitrile concentration in the anaerobic biodegradation. This is in contrast with the maximum acetonitrile concentration of $0.5 \mathrm{gL}^{-1}$ recommended for anaerobic biodegradation with a single species reported by Nagle et al. (1995). In comparison with the performance under aerobic condition (see Fig. 1), the biodegradation performance of acetonitrile under anaerobic condition was only slightly reduced at the concentration of $1 \mathrm{gL}^{-1}$ but much more significantly affected at a higher concentration (i.e., at $\left.2 \mathrm{gL}^{-1}\right)$. Nevertheless, the preliminary results appear to indicate that anaerobic biodegradation of organonitriles with the adapted mixed culture remains to have a good potential, at least in treating relatively low or intermediate organonitrile concentrations. Hence, further study in this aspect may be carried out in the future.

\section{Conclusion}

Suitable microorganisms were successfully enriched from the activated sludge consortium and cultivated with acetonitrile as the only source of carbon and nitrogen. The adapted mixed culture was demonstrated to be very successful in the biodegradation of saturated (acetonitrile), unsaturated (acrylonitrile), and aromatic (benzonitrile) organonitrile compounds. The optimal growth rates of the adapted mixed culture were found to be at $\mathrm{pH} 7.5$ in the $\mathrm{pH}$ range of 3-11 and at $30^{\circ} \mathrm{C}$ in the temperature range of $10-50^{\circ} \mathrm{C}$ studied. Under the normal environmental conditions $\left(25^{\circ} \mathrm{C}\right.$ and $\mathrm{pH}$ 7), the observed maximum growth rate of the culture in this study was in the range of $0.11-0.37 \mathrm{~h}^{-1}$ for the initial acetonitrile concentration range of $500-10,000 \mathrm{mgL}^{-1}$ examined. An average removal rate of $0.083 \mathrm{~g}$ acetonitrile $\mathrm{g}^{-1}-\mathrm{VSS}^{-1}$, 0.0074 g acrylonitrile $^{-1}$-VSS h$^{-1}$ or 0.0029 gbenzonitrile $^{-1}$ $\mathrm{g}^{-1}$ VSS $^{-1}$ was obtained at a batch feed concentration of $2 \mathrm{gL}^{-1}$ acetonitrile, acrylonitrile or benzonitrile, respectively, in the incubations $\left(25^{\circ} \mathrm{C}, \mathrm{pH}\right.$ 7). From the metabolic intermediate- and end-products produced in the processes, the biodegradation of acetonitrile, acrylonitrile or benzonitrile with the adapted mixed culture appeared to involve two different reaction pathways, i.e., nitrile hydratase followed by amidase for acetonitrile and acrylonitrile but only nitrilase for benzonitrile. Preliminary experimental results also show that the adapted mixed culture can degrade acetonitrile fairly well under anaerobic conditions even though the performance was lower than that under aerobic conditions. The study illustrates that the adapted mixed culture had very good adaptation flexibility to different types of organonitriles and had much better degradation performance than many single species reported in the literature. From the practical application point of view, the adapted mixed culture may be expected to have a huge potential for biodegradation of various organonitrile compounds in industrial wastewater.

R E F E R E N C E S

Acharya, A., Desai, A.J., 1997. Studies on utilization of acetonitrile by Rhodococcus erythropolis A10. World J. Microbiol. Biotechnol. 13, 175-178.

Adjei, M.D., Ohta, Y., 1999. Isolation and characterization of a cyanide-utilizing Burkholderia cepacia strain. World J. Microbiol. Biotechnol. 15, 699-704.

Ahmed, A.E., Farooqui, M.Y.H., 1982. Comparative toxicities of aliphatic nitriles. Toxicol. Lett. 12, 157-163.

Alfani, F., Cantarella, M., Spera, A., Viparelli, P., 2001. Operational stability of Brevibacterium imperialis CBS 489-74 nitrile hydratase. J. Mol. Catal. B-Enzym. 11, 687-697.

APHA-AWWA-WEF, 1998. Standard methods for examination of water and wastewater, 20th ed. APHA, AWWA, and WEF, Washington, DC.

Babu, G.R.V., Wolfram, J.H., Marian, J.M., Chapatwala, K.D., 1995. Pseudomonas marginalis: its degradative capability on organic nitriles and amides. Appl. Microbiol. Biotechnol. 43, 739-745.

Banerjee, A., Sharma, R., Banerjee, U.C., 2002. The nitriledegrading enzymes: current status and future prospects. Appl. Microbiol. Biotechnol. 60, 33-44. 
Bhalla, T.C., Kumar, H., 2005. Nocardia globerula NHB-2: a versatile nitrile-degrading organism. Can. J. Microbiol. 51, 705-708.

Bhalla, T.C., Miura, A., Wakamoto, A., Ohba, Y., Furuhashi, K., 1992. Asymmetric hydrolysis of alpha aminonitriles to optically active amino acids by a nitrilase of Rhodococcus rhodochrous PA-34. Appl. Microbiol. Biotechnol. 37, 184-190.

Dias, J.C.T., Rezende, R.P., Linardi, V.R., 2001. Bioconversion of nitriles by Candida guilliermondii CCT 7202 cells immobilized in barium alginate. Appl. Microbiol. Biotechnol. 56, 757-761.

DiGeronimo, M.J., Antoine, A.D., 1976. Metabolism of acetonitrile and propionitrile by Nocardia rhodochrous LL 100-21. Appl. Environ. Microbiol. 31, 900-906.

Endo, I., Watanabe, I., 1989. Nitrile hydratase of Rhodococcus sp. N-774: purification and characterization. FEBS Lett. 243, 61-64.

Håkansson, K., Mattiasson, B., 2002. Microbial degradation of acetonitrile using a suspended carrier biofilm process. Biotechnol. Lett. 24, 287-291.

Håkansson, K., Welander, U., Mattiasson, B., 2005. Degradation of acetonitrile through a sequence of microbial reactors. Water Res. 39, 648-654.

Harper, D.B., 1985. Characterization of a nitrilase from Nocardia sp. (rhodochrous group) NCIB 11215, using $p$-hydroxybenzonitrile as sole carbon source. Int. J. Biochem. 17, 677-683.

Henahan, J.F., Idon, J.D., 1971. Setting the world of nitrle chemistry afire. Chem. Eng. News 49, 16-18.

Johammsen, F.R., Levinskas, G.J., Bertean, P.E., Rodwell, D.E., 1986. Evaluation of teratogenic potential of three aliphatic nitriles in the rat. Fund. Appl. Toxicol. 7, 33-40.

Kao, C.M., Chen, K.F., Liu, J.K., Chou, S.M., Chen, S.C., 2006. Enzymatic degradation of nitriles by Klebsiella oxytoca. Appl. Microbiol. Biotechnol. 71, 228-233.

Kobayashi, M., Yanaka, N., Nagasawa, T., Yamada, H., 1990. Purification and characterization of a novel nitrilase of Rhodococcus rhodochrous $\mathrm{K} 22$ that acts on aliphatic nitriles. J. Bacteriol. 172, 4807-4815.

Langdanhl, B.R., Bisp, P., Ingvorse, K., 1996. Nitrile hydrolysis by Rhodococcus eryhropolis BL1, a acetonitrile-tolerant stain isolated from a marine sediment. Microbiology 142, 145-154.

Lee, C.M., Wang, C.C., 2004. Denitrification with epsilon-caprolactam by acclimated mixed culture and by pure culture of bacteria isolated from polyacrylonitrile fibre manufactures wastewater treatment system. Water Sci. Technol. 49, 341-348.
Lee, S.M., Jung, J.Y., Chung, Y.C., 2000. Measurement of ammonia inhibition of microbial activity in biological wastewater treatment process using dehydrogenase assay. Biotechnol. Lett. 22, 991-994.

Manolov, T., Kristina, H., Benoit, G., 2005. Continuous acetonitrile degradation in a packed-bed bioreactor. Appl. Microbiol. Biotechnol. 66, 567-574.

Muñoz, R., Jacinto, M., Guieysse, B., Mattiasson, B., 2005. Combined carbon and nitrogen removal from acetonitrile using algal-bacterial bioreactors. Appl. Microbiol. Biotechnol. 67, 699-707.

Nagle, N.J., Rivard, C.J., Mohagheghi, A., Philippidis, G., 1995. Bioconversion of cyanide and acetonitrile by a municipalsewage derived anaerobic consortium. In: Hinchee, R.E. (Ed.), Bioremediation of Inorganic. Battelle, Columbus, pp. 71-79.

Nawaz, M.S., Chapatwala, K.D., Wolfram, J.H., 1989. Degradation of acetonitrile by Pseudomonas putida. Appl. Environ. Microbiol. $55,2267-2274$.

Nawaz, M.S., Davis, J.W., Wolfram, J.H., Chapatwala, K.D., 1991. Degradation of organic cyanides by Pseudomonas aeruginosa. Appl. Biochem. Biotechnol. 28 (29), 865-875.

Nawaz, M.S., Heinze, T.M., Cerniglia, C.E., 1992. Metabolism of benzonitrile and butyronitrile by Klebsiella pneumoniae. Appl. Environ. Microbiol. 58, 27-31.

Prasad, S., Sharma, D.R., Bhalla, T.C., 2005. Nitrile- and amidehydrolysing activity in Kluyveromyces thermotolerans MGBY 37. World J. Microbiol. Biotechnol. 21, 1447-1450.

Rezende, R.P., Dias, J., Ferraz, V., Linardi, V., 2000. Metabolism of benzonitrile by Cryptococcus sp. UFMG-Y28. J. Basic Microbiol. 40, 389-392.

Rezende, R.P., Dias, J.C.T., Monteiro, A.S., Carraza, F., Linardi, V.R., 2003. The use of acetonitrile as the sole nitrogen and carbon source by Geotrichum sp. JR1. Braz. J. Microbiol. 34, 117-120.

Shanker, R., Ramakrishna, C., Seth, P.K., 1990. Microbial degradation of acrylamide monomer. Arch. Microbiol. 154, 192-198.

Wang, C.C., Lee, C.M., Chen, L.J., 2004. Removal of nitriles from synthetic wastewater by acrylonitrile utilizing bacteria. J. Environ. Sci. Health Part A Tox. Hazard. Subst. Environ. Eng. 39, 1767-1779.

Yamamoto, K., Ueno, Y., Otsubo, K., Yamane, H., Komatsu, K., Tani, Y., 1992. Efficient conversion of dinitrile to mononitrilemonocarboxylic acid by Corynebacterium sp. C5 cells during tranexamic acid synthesis. J. Ferment. Bioeng. 73, 125-129. 